\title{
Teaching and Learning the Mathematical Exponential and Logarithmic Functions: A Transformation Approach
}

\author{
Sello Makgakga \\ Lecturer: Department of Mathematics Education \\ University of South Africa \\ Email:makgasw@unisa.ac.za \\ Percy Sepeng \\ Senior Lecturer: Department of Mathematics Education \\ University of South Africa \\ Email: sepenp@unisa.ac.za
}

Doi:10.5901/mjss.2013.v4n13p177

\begin{abstract}
This paper discusses the benefits of using a transformation approach in the teaching and learning of exponential and logarithmic functions in a grade 12 mathematics classrooms. The study followed a pre-test - intervention - post-test design with qualitative data informing quantitative data. Data collection strategies included a test (on exponential and logarithmic functions) that was administered to learners before and after the intervention. The intervention strategy was done via the teaching of exponential functions through transformation approach in attempt to reinforce understanding of the concept. A convenience sample of 38 experimental and 40 comparison groups of grade 12 learners participated in the study reported in this article from two different schools to avoid contaminating data collected. The findings indicated that the interventional strategy had an impact on academic performances of the learners. Furthermore, learners had begun to understand and apply the learnt strategy when solving problems related to both exponential and logarithmic functions.
\end{abstract}

\section{Introduction}

Mathematical functions are concepts forms an important part of school mathematics curricula and should be taught with understanding (Curriculum and Assessment Policy Statement, 2012). A function is a "relationship between input values and output values, the output value depends on the input one, and there is only one output for each input value" (Holliday, Cuevas, Moore-Harris, Carter, Marks, Casey, Day, \& Hayek, 2005: 43)). Functions form a major part in school mathematics that is critical to be learned and taught effectively, but are seen as one of the challenging topics for teachers to teach (National Council of Teachers of Mathematics, NCTM, 2010). The study reported in this article explored exponential functions that are expressed in a form $f(x)=c \cdot a^{x}\left(\right.$ or $\left.y=a^{x}\right)$, in which parameter ' $a$ ' is a base which is a positive real number not equal to 1 and parameter $c$ is a function's $y$-intercept (NCTM, 2010; Mousel, 2006).

Exponential functions and logarithmic functions are the two topics that are perceived as connected, and therefore may not be taught in isolation from the other. Logarithmic functions may be viewed as the inverses of exponential functions. Learners appear to experience difficulties in understanding the two types of functions used in the study. Some of the learners struggled to express the exponential equation $y=a^{x}$ into a logarithmic equation, $y=\log _{a} x$. As a result, learners couldn't master a skill of distinguishing them as the rate of growth which is proportional to their values (Bogley \& Robson, 1999). The rate of growth describes the exponential functions decrease as $x$ increases if the base ' $a$ ' is between $0<a<1$, and the function increase as the base $a>1($ NCTM, 2010). There is a need for learners to understand the two functions in order to analyse and interpret problem-solving processes. The interpretation of functions seems to be a problem in the learning of exponential functions. Webber (2002) asserted that exponential and logarithmic functions are important concepts that play a fundamental role in mathematical courses, including calculus, differential equations, and complex analysis. This had shown that there is a need for teachers to teach these functions for understanding as they are mostly used in real-world situations (NCTM, 2010; Mousel, 2006). 


\section{Purpose of the Study}

In the current article, we sought to explore the benefits of teaching exponential functions through transformation and how this technique can assist learners in understanding concept of exponential functions. Although the results reported in this article are of a case study, the findings may go all the way to assist and inform mathematics teachers' practices in the teaching and learning of functions in general. It is against this background that the article aims at assisting the teachers to employ effective pedagogies when teaching exponential and logarithmic functions with understanding by using problem solving skills through a transformation approach. The study's intervention strategy was framed by Polya's (1973) problem solving techniques. Learners were able to identify, analyse, execute and reflect on problems given to them to solve based on exponential function. Essentially, this approach can be piloted by other teachers to teach exponential functions, not these functions per se, but with other functions as well.

\section{Theoretical Perspectives}

Problem solving is regarded as the interaction of cognitive process and mental representation in diverse ways and it is a complex activity (Green \& Gilhoolly, 2005, p. 347). This Problem-solving enabled learners to describe mathematical context in their own way to show understanding of mathematical concepts. In problem solving, Polya suggested four steps approach to problem solving in mathematics and each step requires certain auxiliary skills to work and apply effectively:(a) Identifying and understanding the problem; (b) Devising a plan; (c) Executing the plan; and (d) Looking back (Evaluation). In other words, learners should identify or know the problem to understand it, break the problem into smaller chunks or analyse it, act on a plan or solve a problem, and reflect on it. These steps approach required learners to be in a position to solve problems, such as learners knowing how to draw a simple exponential function, $f(x)=a^{x}(y=$ $\left.a^{x}\right)$ and its inverse $x=a^{y}\left(y=\log _{a} x\right)$ by using a table method. This is an example of a transformation of exponential function into logarithmic function, which is an inverse function; $x$-values become $y$-values in comparing the two functions (Larson \& Hostetler, 1979). For the purposes of this study, we defined the inverse of exponential functions as rotation about $90^{\circ}$ clockwise or anti-clockwise. These types of problems were seen not to be challenging questions for grade 12 learners to solve, but a way of preparing them to solve difficult tasks (Green \& Gilhoolly, 2005; Halpern, 1997; Sternberg \& Benzeev, 2001). However, this was not a case for the study's participants as they struggled to understand and solve exponential functions. Tasks such as analysis and interpretation of exponential and logarithmic functions are regarded as complex ones for learners to solve. These types of tasks appear to require a higher order thinking skills to solve as compared to just drawing a simple exponential function.

\section{Literature Review}

Functions can show the relationships between varying quantities (NCTM, 2010). The function is the relationship between the input variable and the output variable. In the function, the output depends on the input, which means that there is only one output for every input (Holliday, Cuevas, Moore-Harris, Carter, Marks, Carsey, Day, Hayek, 2005). Exponential function is one of the functions that are taught at Further Education and Training. Exponential function is the relationship between the input variables and the output ones, denoted by $f(x)=c a^{x}$ (Mousel, 2006; Webber, 2002). This function is characterized by the rate of change that is proportional to the value of a function (NCTM, 2010; Bogley \& Robson, 1999). Exponential function has a property that whenever the input is increased by 1 unit, the output is multiplied by a constant factor. It connects multiplication to addition through the equation $a^{b+c}=(a)^{b}(a)^{c}$. NCTM (2010) and Mousel (2006) argued that exponential function describes many real-world problems that involve growth or decay.

Webber (2002) indicated that learners understand exponential and logarithmic functions through exponentiation as an action and process, exponential expressions are the results of the process and generalisation. Learners being able to view exponentiation as action and process, are the ones who can compute $b^{x}$ as $b x$ times and when they repeat the action and reflect upon it, they interiorise that action as the process (Dubinsky, 1991). Terms such as $2^{4}$ can be interpreted as an external prompt for the student to compute $2 \times 2 \times 2 \times 2$, which is a product of four factors of 2 . Research indicates that students are not capable of viewing $2^{4}$ in this way (e.g. Sfard, 1991). In generalization, learners have a full understanding of exponential functions which involve interpreting situations where a number to be evaluated is a fraction (Webber, 2002).

Mousel (2006) explained exponential functions by using table method, of $f(x)=2^{x}$. The function was then transformed into $f(x)=2^{x-1}$ and also used the table method to explain the shifting of the graph from the function $f(x)=2^{x}$, 
which was called the horizontal shift to the right. Furthermore, the reflection of the function about the $x$-axis was explained as the one with the horizontal asymptote. Moreover, Mousel discussed the reflection of $f(x)=2^{x}$ about the line $y=x$, which gives the logarithmic function $y=\log _{2} x$, the inverse of exponential function. The line $y=x$ is considered as the perpendicular bisector of the line segments created by joining inverse points from the logarithmic and exponential functions (Allendoerfer, Oakley, \& Kerr, 1977). Comparing the two functions, we would say that a logarithm is an exponent (Larson \& Hostetler, 1979).

Literature review is also done on transformation. Transformation is a unifying topic in the middle school geometry curricular (Lappan \& Even, 1988; Wu, 2005). Seago, Nikula, Matssa and Jacobs (2012) used transformation approach to teach geometry concept, similarity. Scholars also found that teaching conceptually based understanding of geometric transformations which includes translation, rotation, reflection and dilation is likely to have a profound effect on learners' ability to learn a wide range of mathematical topics (Ada \& Kurtulus, 2010). Materials such as video cases were also developed to deepen the middle school teachers' knowledge of geometric transformations and supporting their understanding of how a transformation approach in mathematical similarity can be applied in the classroom (Borko, Koellner, Jacobs \& Seago, 2011; Seago, Driscoll \& Jacobs, 2011). Those materials are developed for Learning and Teaching Geometry (LTG) teacher professional development (PD). The LTG PD guides teachers to move beyond conceptualising similarity as a numerical relationship between two discrete figures. Henceforth, also guides teachers to understand a precise conception of similar figures from transformation-based perspectives. Those materials also have a significant growth in teachers' mathematical understanding of this challenging content (Seago et al, 2012).

Bansilal and Naidoo (2012) concentrated on the use of visualisation and analytic strategies by grade 12 learners when working with problem based on transformation geometry. Bansilal and Naidoo found that most of learners performed treatments in analytic mode when responding to questions on transformations. Furthermore, they showed a limited movement across the two modes which are essential for deepening understanding. Duval (2003) emphasised that conversion type activities which involve movement across different representations are essential for an enhancing understanding. Zaskies, Dautermann, \& Dubinsky (1996) contend that even though both visual and analytical strategies may be available to learners, they 'often have difficulty making connections between them'.

Smith (2009) argues that learning functions transformations help learners better understand each function's behaviour and relationships. Studies revealed that learning the function concept is concept with many high performing undergraduates possessing weak function understandings (Carlson, 1998; Oerhtman et al. 2008). Learners' understanding of transformation of graphs is very limited (Smith, 2009). Transformation of graphs has been a major component of many levels of algebra as well as of more advanced courses in mathematics (Ninnes, Barness-Holmes, 2006). Smith used idea of co-variation to reason about transformation of functions. By employing the co-variation reasoning (e.g. coordinating an image of two varying quantities and attending to how they change in relation to each other), the learners are able to transform the image and reason about values of various parameters as the configuration changes (Oerhtman, 2008). He concluded that function transformation has a different approach than the textbook. Learners had grown from total of procedural approach to trying to understand and reason about how a function can be transformed.

\section{Methodology}

The study explored the transformation approach as an intervention to teach and learn exponential and logarithmic functions. A pre-test, which was exactly the same with a post-test, consisted of items based on exponential and logarithmic functions were administered to 38 experimental and 40 comparison grade 12 learners from two different schools for the purpose of preventing the contamination of the results (McMillan \& Schumacher, 2010). The test included items that included drawing exponential functions, analysing and interpreting exponential functions. The pre-test was administered before the study and the post-test was administered after the intervention for both comparison and experimental groups. Interventions were done on these two functions used experimental group with the aim of exploring the transformation approach in teaching and learning exponential and logarithmic functions. The lessons were conducted over a period of 30 days to complete all the concepts of functions and both groups were then given a post-test immediately after the intervention.

\section{Ethical Considerations}

The permission letter for the researcher to conduct the study was submitted to the two principals of the schools where 
the researcher was attached and the approval of the application was done by the School Management Team. The letter entailed the application for grade 12 learners to participate in the study as it was action research, teaching and learning exponential functions. Learners were informed about the study, reasons and purpose of the research also discussed with them. The letter also discussed and defined informed consent, the right to withdraw without being prejudice and confidentiality as advised by (Bokdan \& Biklen, 2003). As the study was action research, other teachers were not included and learners' names were used as pseudonyms to protect their identity (Johnson \& Christensen, 2004).

\section{Intervention}

Learners were given a pre-test, both 38 experimental group and 40 comparison groups in the same school. Transformation approach was used in teaching the experimental group exponential functions and logarithmic functions. This approach was used to reinforce understanding of the relationship of exponential and logarithmic functions, as well as analysing and interpreting any given problem related to them. The definition of exponential function as a function of a basic form $f(x)=c a^{2}$, where parameter $a$ is a base which is a positive real number $(a>0$ and $a \neq 0)$ and $c$ is a function $y$ intercept (NCTM, 2010; Mousel, 2006). It was highlighted to learners not to intertwine the exponential function $f(x)=a^{2}$ and the parabola $f(x)=x^{2}$. Learners were given the functions, $f(x)=2^{x}, f(x)=3^{-x}$ and $f(x)=4^{x}$ to discuss in groups. They were also asked to discuss their domains, ranges and determined whether the functions were increasing or decreasing functions.

Reflection, translation and rotation were the three forms of transformation that were used to explain exponential and logarithmic functions. The function $f(x)=2^{x}$ was used to explain the three forms of transformation with the horizontal axis as the asymptote. The function was reflected about the $y$-axis and the $x$-axis. The reflection of $f(x)=2^{x}$ about the $y$ axis, the value of $x$ changed the sign and the new function was $f(x)=2^{-x}$ with their $y$-intercept as $(0,1)$. The other reflection about the $x$-axis was of the function $f(x)=-2^{x}$ which changed the sign of the $y$-value and with the $y$-intercept $(0$, -1). The reflection of the function $f(x)=2^{-x}$ about the $x$-axis was $f(x)=-2^{-x}$. The researcher used his body to demonstrate the reflections of these different functions mentioned. The first two functions, two hands were raised curved up and the last two curved down as they reflected about the body standing upright. The body was used as the $y$-axis and shoulders as the $x$-axis, and hands were used to demonstrate the graphs.

The function $f(x)=2^{x}$ was also translated up and down, further away from the y-axis and closer to $y$-axis. In translating up and down, the functions $f(x)=a^{x}+q$ and $f(x)=a^{x}-q, q$ was the point of asymptote. Learners were asked to discuss the functions $f(x)=2^{x}+3$ and $f(x)=2^{x}-3$ to observe their movements. The researcher moved his body with the hands curved up, up and down to demonstrate the movement of the two functions. Their $y$-intercepts were $(0,4)$ and $(0,-2)$ respectively according to their functions. Translation of the function $f(x)=2^{x}$ was used side-way, the graph moved to left and to the right. This was the function $f(x)=a^{x-c}$ and $f(x)=a^{x+c}$, the first one was explained that its movement was to the right and the second one to the left. The functions $f(x)=2^{x-1}$ and $f(x)=2^{x+1}$ with respect to $f(x)=2^{x}$ were given to learner for discussion. Learners were asked to use tables to discuss the translation of these functions, starting with the original function that they use to compare the movement. The table that follows explained the translation of the functions well, learners understood how they moved when they subtract and add 1.

$y=2^{x}$
\begin{tabular}{|c|c|c|c|c|c|c|c|}
\hline$x$ & -3 & -2 & -1 & 0 & 1 & 2 & 3 \\
\hline$y$ & $\frac{1}{8}$ & $\frac{1}{4}$ & $\frac{1}{2}$ & 1 & 2 & 4 & 8 \\
\hline
\end{tabular}

y $=2^{x-1}$
\begin{tabular}{|c|c|c|c|c|c|c|c|}
\hline$x$ & -3 & -2 & -1 & 0 & 1 & 2 & 3 \\
\hline$y$ & $\frac{1}{16}$ & $\frac{1}{8}$ & $\frac{1}{4}$ & $\frac{1}{2}$ & 1 & 2 & 4 \\
\hline
\end{tabular}

$y=2^{x+1}$
\begin{tabular}{|l|l|l|l|l|l|l|l|}
\hline$x$ & -3 & -2 & -1 & 0 & 1 & 2 & 3 \\
\hline$y$ & $\frac{1}{4}$ & $\frac{1}{2}$ & 1 & 2 & 4 & 8 & 16 \\
\hline
\end{tabular}

Initially the researcher raised his right hand curved up and showed learners how the graphs translated. The hand was moved further away from the head which demonstrated the second one and again moved the hand closer to the head to demonstrate the third. The second graph explained that the graph moved to the right and the third one moved to the left. Even the points in the tables explained the shifting of the points clearly.

The inverse function of $f(x)=2^{x}$ was explained as the rotation either about $90^{\circ}$ clockwise or anti-clockwise or the reflection about the line $y=x$ or $y=-x$. It was explained that in rotation process, $x$ values become $y$-values and viceversa. Learners were given exponential equations to change them into logarithmic form, $y=a^{x}, b=d^{c}$ and $e=j^{k}$ in their discussion groups. The researcher asked learners to draw the rotation of the functions, $f(x)=2^{x}, f(x)=2^{-x}$ and $f(x)=-2^{-x}$ about $90^{\circ}$ clock-wise, and they were also reminded that the first graph was an increasing function approached the negative $\mathrm{x}$-axis and the second one was a decreasing one approaching the positive $\mathrm{x}$-axis with the horizontal asymptote. 
In the discussion, the researcher used his body once more. The first one the researcher twisted the right hand curved up, the second one the hand was twisted curved down and the last one the left hand was twisted curved up with their vertical asymptotes. The first two functions had their $y$-intercepts $(0,1)$ initially and changed to $x$-intercepts $(1,0)$, and while the last one changed $y$-intercepts from $(0,-1)$ to $(-1,0)$.

\section{Results}

A pre-test was administered to 38 comparison and 40 experimental groups of learners for the researcher to understand the learners' level of understanding exponential and logarithmic functions. Learners' outcomes of the pre-test are:

\subsection{Pre-test results}

\begin{tabular}{cccccc}
\hline Groups & No wrote & No pass & $\%$ pass & No fail & $\%$ fail \\
\hline Experimental & 38 & 12 & $31.6 \%$ & 26 & $68.4 \%$ \\
Comparison & 40 & 15 & $37.5 \%$ & 25 & $62.5 \%$ \\
\hline
\end{tabular}

\subsection{Percentage analysis}

\begin{tabular}{ccccccc}
\hline Groups & $0-39 \%$ & $40-49 \%$ & $50-59 \%$ & $60-69 \%$ & $70-79 \%$ & $80-100 \%$ \\
\hline Experimental & 33 & 3 & 2 & - & - & - \\
comparison & 29 & 6 & 3 & 2 & - & - \\
\hline
\end{tabular}

The results confirm that most of the learners both on experimental and comparison groups performed badly in the pretest. Out of 38 in the experimental group, 25 learners managed to draw simple exponential functions, 8 have successfully drawn the functions like $f(x)=a^{x}+q$, but only 2 learners managed to answer questions based on analysis and interpretation of exponential functions. Out of 40 learners in the comparison group, 27 learners managed to draw simple exponential functions, 11 have successfully drawn the functions like $f(x)=a^{x}+q$, and only four learners managed to answer questions based on analysis and interpretation of exponential functions. The highest mark is $52 \%$ and the lowest mark was $11 \%$ in the experimental group which gave the range of $41 \%$. This has drawn the researcher's attention and prompted one to conduct this study to limit the challenges learners had in exponential functions. The overall results had shown that learners had challenges in dealing with exponential and logarithmic functions and this had prompted the researcher to explore the transformation approach to improve learners' academic achievement in this concept. Learners in the experimental and comparison groups were given a post-test evaluation after the interventions with the experimental group.

Experimental and comparison groups of learners were given a post-test evaluation to understand learners' problem solving skills provided to them in teaching transformation of exponential function used by the researcher. Results were as follows (learners A - pre-test and learners B- post-test):

No wrote (A): 40 No Pass: $14 \%$ Pass: 35\% No Fail: $26 \%$ Fail: $65 \%$

No wrote (B): 38 No Pass: 34 \% Pass: 89.5\% No Fail: 26 \% Fail: 10.5\%

\begin{tabular}{|c|c|c|c|c|c|c|c|}
\hline Level & 1 & 2 & 3 & 4 & 5 & 6 & 7 \\
\hline Comparison (A) & 26 & 7 & 5 & 2 & - & - & - \\
\hline Experimental (B) & 4 & 3 & 8 & 10 & 6 & 4 & 3 \\
\hline
\end{tabular}

\subsection{Pre-Test and Post-Test Results}

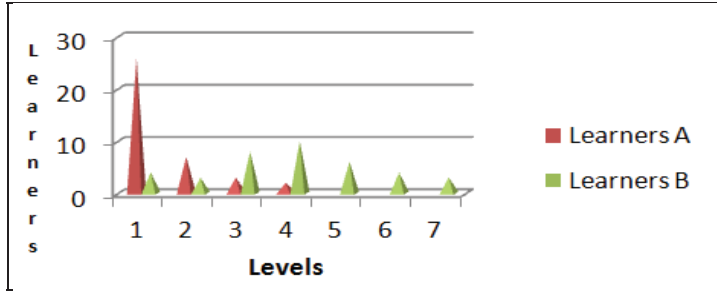


The results showed that most of the learners performed well in the test as only four learners failed, as compared to the pre-test results. Most of the learners performed well in the test which gave the mean percentage of $89.5 \%$ and range of approximately 10.5. The range of the post-test results was much better compared to the pre-test average mark of $41 \%$. The table showed that 4 learners from experimental group obtained between $0 \%-29 \%$ as compared to the 29 learners in the comparison group, 3 between $30 \%$ - 39\% as compared to 7, 8 between $40 \%$ - 49\% as compared to 3, 10 between $50 \%-59 \%$ as compared to 2, 6 between $60 \%-69 \%$, 4 between $70 \%-79 \%$ and 3 between $80 \%-100 \%$. This has indicated to the researcher that the number of learners passed increased by 22 and their mean percentage increased by 57. $9 \%$.

The post-test evaluation was out of 60 marks in which question 1 comprised 21 marks, question 2 has 15 marks and question 3 has 24 marks. The table below provides an overview of item analysis of the test and how learners performed in each question.

\subsection{Post-Test Evaluation}

\begin{tabular}{|c|l|c|c|c|}
\hline Question & \multicolumn{1}{|c|}{ Description } & Marks & $\begin{array}{c}\text { No of } \\
\text { Learners/question }\end{array}$ & $\begin{array}{c}\text { \% obtained by } \\
\text { learners }\end{array}$ \\
\hline 1.1 & Drawings & 9 & 38 & $100 \%$ \\
\hline 1.2 & Reflection of graphs and equations of the functions & 12 & 34 & $89.5 \%$ \\
\hline 2.1 & Translation of $y=3^{x}$ to $y=3^{x}+1$ & 4 & 36 & $94.7 \%$ \\
\hline 2.2 & Translation of $y=\left(\frac{1}{2}\right) \cdot \cdot^{x-3}$ & 4 & 35 & $92.1 \%$ \\
\hline 2.3 & Rotation of $y=5^{x}$ (Inverse Function) & 7 & 33 & $86.8 \%$ \\
\hline 3.1 & $\begin{array}{l}\text { Analysis and interpretation of } y=a^{x} \text { with a point }\left(1, \frac{1}{2}\right) \text { and } \\
\text { sub-questions 3.1.1-3.1.6 }\end{array}$ & 14 & 27 & $71.1 \%$ \\
\hline 3.2 & $\begin{array}{l}\text { Analysis and interpretation of } y=\log _{2} x \text { with sub-questions } \\
3.2 .1-3.2 .5\end{array}$ & 10 & 25 & 65.85 \\
\hline
\end{tabular}

Question 1 comprised drawing of the functions $y=3^{x}, y=(1 / 4)^{x}$ and $y=-2^{x}$ respectively and their reflections. All the learners managed to draw all the functions correctly but four of them did not draw the reflection of those functions. Three of these four learners have successfully written the equations of the reflected functions as $y=3^{-x}, y=(1 / 4)^{-x}$ and $y=-2^{-x}$. The challenge they experienced was on drawing the graphs of the reflected functions. These learners did not understand how the reflection about the $y$-axis which resulted in giving wrong answers on this question. They gave the correct equations of the reflected functions and failed to draw them. They should at least have used table method to draw those functions as they had challenges in just drawing them by reflection. It was understood the variable $x$ should change the sign to be negative. In question 2, questions were based on translation or shifting of the graph of $y=3^{x}$ to $y=3^{x}+1$.

Most of the learners had understood how to translate the function $y=3^{x}$ to $y=3^{x}+1$ which was question 2.1 but two of them did not do well in this question. They did not answer the question at all which indicated to the researcher that they didn't understand how to translate the function. These learners had intertwined the behaviour of exponential functions and linear functions. In linear functions, 1 would be correct if they said it was the $y$-intercept but not in exponential functions. They treated the function as $y=3 x+1$ which was incorrect. They thought that the constant 1 was the same as in exponential functions which was incorrect to marry the two constants. Three other learners failed to draw the graph of the function $y=(1 / 2)^{x-1}$ in (2.2) which was the translation of the function $y=(1 / 2)^{x}$ to the right. The remained learners understood that if the exponent $x$ subtracts any integer except 0 , then the graph shifts to the right.

Question 2.3 discussed the rotation or the inverse of the function $y=5^{x}$ about $90^{\circ}$ clock-wise direction as explained during the lesson presentations. At least five learners failed to draw the inverse function and the equation of the function. These learners have drawn the graph of the function of $y=5^{x}$ instead of $y=\log _{5} x$ which was the rotation of the original function. They forgot that in rotation the input values become the output values, i.e $x$-becomes $y$ and $y$ becomes $x$. Thirty-three learners had successfully drawn the graph of the function $y=\log _{5} x$, they interchanged the values of $x$ and $y$. They understood that the original function cut the $y$-axis at $(0,1)$ and then interchanged them where the new graph cut at $x$-axis at $(1,0)$. The explanation of the line approaching the negative $x$-axis assisted them to draw their graph as it approached the negative $y$-axis. It was understood that when the function increases, it approaches the negative $x$-axis and its rotation approach the negative $y$-axis as explained in the classroom discussion.

Learners were given question 3.1 and 3.2 to analyse and interpret them respectively. In these questions, 11 and 13 learners did not do well in these questions respectively. The function of question 3.1 was $h(x)=a^{x}$ and had sub- 
questions as explaining the coordinates of the $y$-intercept, calculating the value of $a$, finding the equation of the rotated function (inverse), sketching the rotated function, reading the values of $x$ from the graph and determining the value of $x$ when the two graphs are equal. There were 24 learners who explained why at the $y$-axis were $(0,1)$, and said any number to exponent zero is 1 except zero. They also determined the value of a as 2 because they substituted the $x$ by -1 and $y$ by $1 / 2$ which gave the answer as 2 . The rotation of the function $y=\log _{2} x$ was determined and sketched. In interviewing them, they explained that by looking at the given function, it was an increasing function and approached the negative $x$-axis cut the $y$-axis at $(0,1)$. The rotated function cut the $x$-axis at $(1,0)$ approached the negative $y$-axis. This had shown that learners have understood the teaching and learning of exponential functions through transformation approach. Three learners answered questions up to 3.5 where they had to read from the graph the values of $x$ for which $\log _{2} x>-1$ which was $x>0.5$ without any calculations. The question required learners to show the values of $x$ from the graph where they could give the output values greater than -1 . One learner answered all the questions correctly, in which question 3.6 expected them to determine the value of $x$ for which (100). $3^{x}=2^{x}$. This girl had successfully simplified the equation and had also introduced logarithms both sides to find the value of $x$ as 11 . 36. At least eleven learners did not analyse and interpret the graph well, but managed to answer questions based on the $y$-intercept and finding the value of a. The performance was much better as compared to the previous performance of analysing and interpreting exponential functions.

Learners have also responded to a question based on the graph $f(x)=-\log _{2} x$. The question expected them to write down the domain of $f$, the inverse function in the form $y=\ldots$ and the equation of the asymptote of $f^{1}$. They were also asked to explain how, using the graph of $f$, they would sketch the graph of $g(x)=\log _{2} x$ and $h(x)=2^{-x}-5$. From a question on calculating the value of $x$ for which $\log _{2} x<3$ by using the graph, about twenty out of thirty eight learners correctly computed a domain of $f$ as $x €(0, \infty)$ or $x>0$. They also found the inverse of the function as $y=2^{-x}$ or $y=(1 / 2)^{x}$ and the equation of the asymptote of $f^{-1}$ as $y=0$ or the horizontal $x$-axis. Learners explained that when graph cuts the $y$-axis at $(0$, 1) either increasing or decreasing functions, the asymptote of the graph is $y=0$ or horizontal $x$-axis. This had shown that learners were able to analyse and interpret the graph given to answer questions followed and reflected what they have done. It was also explained that the transformation of the function $g(x)=\log _{2} x$ was the reflection of the function $f(x)=$ $\log _{2} x$ about the $x$-axis. The function $h(x)=2^{-x}-5$ was described as the rotation of the graph $y=-\log _{2} x$ about $90^{0}$ anticlockwise direction and translated five units downwards. Learners had a good understanding of transformation of exponential functions and used it to solve problems related to these functions. Five learners successfully solved the values of $x$ for which $\log _{2} x>3$ and they found those values of $x$ as $0<x<8$ or $x €(0$, 8). Only thirteen learners of the group attempted to answer some of the questions from 3.1.1 to 3.1.4 and produced a minimum mark of $3 \%$.

\section{Results and Discussion}

This study used transformation approach in teaching and learning exponential and logarithmic functions. Experimental group of learners understood the relationships between two quantities, input variables and output variable (NCTM, 2010; Mousel, 2006; Webber, 2002). Most of the learners had effectively drawn exponential functions and their transformed ones as compared to the comparison group where most of them did not do that much. Furthermore, the comparison group only managed to respond to questions up to question 2 , and some not all the questions. This had revealed that the concept was still a problem to these learners.

Mousel (2006) discussed how to understand exponential and logarithmic functions by using table method, transformation approach was used but not deeply. This study went further more to use transformation approach to teach and learn these functions and had an impact in experimental group's academic achievement. Moreover, the study used the researcher as a resource to reinforce understanding of transformation of functions in the form of reflections, translations and rotations which were not discussed in details in the literature. Webber (2002) argued about exponentiation as action and process, exponential expressions as process and generalisation. Webber's study did not add much to this study as it focused mainly on understanding exponential functions by using the stated factors. Therefore, this study went further discussed exponential and logarithmic functions by using transformation approach, such rotation, translations and reflections. Furthermore, the intervention strategy concentrated on problem-solving of the two functions.

Problem-solving skills in the two functions were demonstrated by the experimental group as they interpreted and analysed questions that required their higher order thinking. Moreover, experimental group performed much better as compared to comparison group in questions required higher order thinking. The experimental group followed Polya's four steps of problem solving; identifying and understanding the problem, analysing, execution and evaluation. The 
transformation of exponential functions were interpreted and correctly answered in problem solving in the experimental group. In this regard, learners were prepared to solve difficult problems in exponential and logarithmic functions (Green \& Gilhoolly, 2005; Halpern, 1997; Sternberg \& Benzeev, 2001). Teaching and learning using transformation approach played a fundamental role in exponential and logarithmic functions as experimental group performed much better as compared to the comparison group performance. This had revealed that learners in the comparison group still had challenges in dealing with exponential and logarithmic functions.

\section{Conclusion}

The use of transformation approach to teach and learn exponential function had played a fundamental role in this study. The approach was effective as it made learners to improve their academic performance in dealing with exponential and logarithmic functions. The experimental group performed much better before the intervention as compared to the pre-test results and that of the comparison group. The findings of this small-scaled study seemed to suggest that learners in the experimental group were able to analyse and interpret any given exponential and logarithmic functions in order to solve the related problems. The overall results of the study reported in this article revealed that this approach appeared to be useful and could be used to teach any topic related to functions.

\section{References}

Ada, T. \& Kurtulus, A. (2010). Students' misconceptions and errors in transformation geometry. International Journal of Mathematical Education in Science and Technology, 41(7), 901-909.Allendoerfer, C., Oakley, C., \& Kerr, D.Jr. (1977). Elementary functions. McGraw-Hill, Inc.

Bansilal, S. \& Naidoo, J. (2012). Learners engaging with transformation. South African Journal of Education, vol. 32: 26-39, EASA.

Bogdan, R.C. \& Biklen, S.A. (2003). Qualitative Research for Education: An Introduction to Theories and Methods (4th ed.). Boston: Allyn \& Bacon.

Bogley, W. \& Robson, Robby (1999). http://oregonstate.edu/instruct/mth251/cq/Fieldguide/exponential/lesson.html.

Borko, H., Koellner, K., Jacobs, J. \& Seago, N. (2010). Using video representations of teaching in practice-based professional development programs. Zentralblatt für Didaktik der Mathematik: International Reviews on Mathematical Education, 43(1), 175187.

Carlson M. P. A Study of Second Semester Calculus Students' Function Conceptions. Arizona State University.

Duval R 2002. The cognitive analysis of problems of comprehension in the learning of mathematics. Mediterranean Journal for Research in Mathematics Education, 1:1-16.

Green, A.J.K., \& Gilhoolly, K. (2005). Problem-solving. In Braisby, N. AND Gelatly, A. (Eds.). Cognitive Psychology. Oxford: Oxford University Press.

Halpern, D. F. (1997). Critical Thinking Across the Curriculum: A brief edition of thoughts and knowledge. London: Lawrence Erlbaum Associates.

Holliday, B., Cuevas, G. J., Moore-Harris, B., Carter, J. A., Marks, D., Casey, R. M., Day, R., Hayek, L. M. (2005). Algebra 1. New York: Glencoe/McGraw Hill.

Kennedy, M. (1998). Form and substance in in-service teacher education (Vol. 13). Arlington VA: National Science foundation.

Larson, R. \& Hostetler, R. (1979). Calculus with analytic geometry. Lexington, MA.: D. C. Heath and company.

Luneta, K. (2003). Developing and Piloting a Mentor Training Programme for Teaching Supervision for Mathematics Primary School Teachers. Johannesburg: University of Witwatersrand.

Luneta, K. (2006). Error Discourse in Mathematics and Science: Perspectives of Students' Misconceptions. Johannesburg: University of Johannesburg.

McMillan, J. H. \& Schumacher, S. (2010). Research in Education. Evidence-Based Inquiry. Pearson Education, Inc., Upper Saddle River, New Jersey.

Mills, G. (2006). Action Research: A Guide for the Teacher Researcher (3rd ed.). Columbus, OH: Merrill Prentice.

Mousel, S. A. (2006). The Exponential Function Expository Paper.

National Council of Teachers of Mathematics (NCTM, 2010). Developing Essential understanding of Functions. The National council of Teachers of Mathematics: Reston.

Ninness C. (2005). Transformations of mathematical and stimulus functions, In Barnes- Holmes D., Rumph R. (Eds), CBMS issues in Journal of Applied Behavior Analysis. 2006,36, 299-321.

Oehrtman, M. C., Carlson, M. P., \& Thompson, P. W. (2008). Foundational Reasoning Abilities that Promote Coherence in Students' Function Understanding. Making the Connection: Research and Practice in Undergraduate Mathematics (pp. 27 - 42). MAA Notes. Mathematical Association of America.

Polya, G. (1973). How to solve it: A new aspect of mathematical method. New Jersey: Prince University Press.

Seago, N., Driscoll, M., \& Jacobs, J. (2010). Transforming middle school geometry: Professional development materials that support the teaching and learning of similarity. Middle Grades Research Journal, 5(4), 199-211. 
Seago, N., Nikula, J., Matassa, M., \& Jacobs, J. (2012). A transformations-based approach to learning and teaching similarity. $12^{\text {th }}$ International Congress on Mathematical Education, COEX, Seoul, Korea.

Smith, H. H. (2009). Teaching and learning function transformation. Research Paper for Masters Degree. Arizona State University.

Thompson P. W. (1994). Students, Functions, and the Undergraduate Curriculum. In E.Dubinsky, A. H. Schoenfeld, \& J. J. Kaput (Eds.), Research in Collegiate Mathematics Education, 1. Issues in Mathematics Education Vol. 4, pp. 21-44.

Oehrtman, M. C., Carlson, M. P., \& Thompson, P. W. (2008). Foundational Reasoning Abilities that Promote Coherence in Students' Function Understanding. Making the Connection: Research and Practice in Undergraduate Mathematics (pp. 27 - 42). MAA Notes. Mathematical Association of America

Webber, K. (2002).Students' Understanding of Exponential and Logarithmic Function. Murray State University: USA.

Zazkis R, Dautermann J \& Dubinsky E 1996. Coordinating visual and analytical strategies. A study of students' understanding of the group D4. Journal for Research in Mathematics Education, 27:435-457. 
